\title{
Finite Element Code for Multidimensional Numerical Analysis of Squirrel-Cage Induction Motor
}

\author{
M.G. Persova, Yu.G. Soloveichik, N.V. Kondratyev \\ Department of Applied Mathematics \\ Novosibirsk State Technical University (NSTU) \\ Novosibirsk, Russia \\ persova@ami.nstu.ru
}

\author{
Z.S. Temlyakova, A.A. Temlyakov \\ Department of Electromechanics \\ Novosibirsk State Technical University (NSTU) \\ Novosibirsk, Russia \\ temlikova@edu.nstu.ru
}

\begin{abstract}
The paper is devoted to the development of specialized software for simulating the electrodynamic processes in induction motors. This software includes both the graphical interface and the computational modules based on quasi-3D finite element modeling of electrodynamic processes as well as 3D finite element modeling of the magnetic field in order to take into account the end-zones influence on the values of the magnetic fluxes and rotating torque. The numerical data are compared with the experimental data. The results of the simulation of transport vehicle acceleration for different modes are presented. The proposed software allows the engineers to perform the quasi3D modeling of electrodynamic processes in the induction motor even if they are not experts in numerical methods.
\end{abstract}

Keywords - squirrel-cage induction motor; finite element modeling; electrodynamic process

\section{INTRODUCTION}

The application of modern computational modeling makes it possible to simulate induction motor performance almost completely and with high accuracy. For this purpose, it is necessary to do the following. Firstly, it is required of the corresponding software of computational modeling to be based on the calculations of multidimensional electromagnetic fields in the induction motor structure and correctly take into account the specificity of the electric circuit. Secondly, the electromagnetic force should be calculated using the distribution of the electromagnetic field in the 3D structure of the induction motor. This makes it possible to adequately calculate the rotor speed, perform the corresponding rotations of the rotor, and obtain the important characteristics of the induction motor: currents, power, losses, and etc.

The finite element method is widely used for the analysis of the electromagnetic [1-3] and heat [4-7] processes in the induction motor. For this purpose, both $2 \mathrm{D}$ and $3 \mathrm{D}$ techniques of modeling are applied. One of the most recent papers in the field [8] considers systematically coupling 2D and axisymmetric finite-elements models. Paper [9] is devoted to the 3D analysis of influence of stator winding asymmetry on axial flux. Paper [10] is devoted to the comparison of the approaches based on $2 \mathrm{D}$ and $3 \mathrm{D}$ modeling of the magnetic field for single-phase shaded pole induction motors.
In this paper, for numerical analysis of a squirrel-cage induction motor, the software based on quasi-3D modeling of electrodynamic processes as well as 3D modeling of the magnetic field is proposed. The latter is used in order to take into account the end-zones influence on the values of the magnetic fluxes and rotating torque.

The design of the induction motor structure and generation of the corresponding finite element mesh for modeling the electrodynamic processes are the main and time-consuming stages of the whole computational process. At the same time, on the one hand, the induction motor geometry is quite complex, if the most common finite element codes are used. But, on the other hand, it is specified, i.e. the induction motors consist of a particular set of structural parts, which can be selected from the corresponding «slots library» indicating their specific parameters.

In this paper, a computer system for designing the squirrelcage induction motors is proposed. This system includes both the graphical interface and the computational modules, which allow the engineers to perform the quasi-3D modeling of electrodynamic processes in the induction motor (even if they are not experts in numerical methods).

\section{Mathematical Models AND Software}

\section{A. Quasi-3D Mathematical Model}

The proposed software is based on the mathematical model which is represented by the hybrid system of the nonlinear partial differential, integral, and algebraic equations and has the following form [11]:

$$
\begin{aligned}
& -\nabla\left(\frac{1}{\mu B} \nabla A_{z}\right)=\sigma E_{z}, \\
& \frac{1}{n_{b} l_{b}}-V_{j-1}+2 V_{j}-V_{j+1}+\frac{l}{l_{b} S^{\mathrm{w}}} Q_{j}-Q_{j-1}=0, \\
& j=1 \ldots N^{p h} N^{b r} N^{\mathrm{w}}-1, \quad j \neq m N^{\mathrm{w}},
\end{aligned}
$$




$$
\begin{aligned}
& j \neq m-1 \quad N^{\mathrm{w}}+1, \quad m= 1 \ldots N^{p h} N^{b r}, \\
&-\sigma_{p}^{r} \frac{S_{p}^{r}}{d_{p}^{\text {cT }}} V_{p, k-1}+2 V_{p, k}\left(\sigma_{p}^{r} \frac{S_{p}^{r}}{d_{p}^{b}}+\sigma_{p}^{b} \frac{S_{p}^{b}}{l}\right)- \\
& \quad-\sigma_{p}^{b} \frac{S_{p}^{r}}{d_{p}^{b}} V_{p, k+1}+\sigma_{p}^{b} G_{p, k}=0 .
\end{aligned}
$$

In these equations, the following notations are used:

$\mu$ - the magnetic permeability which depends, in the general case, on the absolute value of the vector of magnetic induction $B=\sqrt{B_{x}^{2}+B_{y}^{2}}$;

$A_{z}$ - the $z$-component of the vector potential of the magnetic field, with the help of which the components of the magnetic field induction are defined as $B_{x}=\frac{\partial A_{z}}{\partial y}$ and $B_{y}=-\frac{\partial A_{z}}{\partial x}$

$\sigma-$ the electrical conductivity;

$E_{z}$ - the $z$-component of the vector of the electric field strength which is defined by the time derivative of $A_{z}$ and difference of the corresponding values of the potentials $V_{j}$ or $V_{p, k}$

$V_{j}$ - the scalar electric potential at the points where the coils of the parallel branch of the stator winding are connected;

$V_{p, k}$ - the scalar electric potential at the point where the $k$-th rod of the squirrel cage with a number $p$ is connected to the corresponding short-circuited ring in rotor;

$S^{\mathrm{w}}$ - the area of the cross section of the effective phase conductors in the stator winding;

$n_{b}-$ the number of turns in one coil;

$l_{b}-$ the length of the turn;

$l$ - the length of the motor;

$N^{p h}$ - the number of phases;

$N^{b r}$ - the number of branches in the phase connected in parallel;

$N^{\mathrm{w}}$ - the number of the coils connected in series in a parallel branch;

$S_{p}^{b}-$ the area of the cross section of a rod in the squirrel cage;

$\sigma_{p}^{b}-$ the electric conductivity of the material of the rod; $d_{p}^{b}$ - the distance between the centers of the rods;

$S_{p}^{r}-$ the area of the cross section of the short-circuiting ring;

$\sigma_{p}^{r}$ - the electric conductivity of the material of the shortcircuiting ring;

$Q_{j}$ - the time derivative of the flux of magnetic induction through the $j$-th coil;

$G_{p, k}$ - the inductive component of the current in the $p$-th rod of the $k$-th squirrel cage;

$\nabla$ - Hamiltonian operator.

$Q_{j}$ and $G_{p, k}$ are defined by the time derivative of potential $A_{z}$ in the form:

$$
Q_{j}=\int_{\Omega_{j}^{w+}} \frac{\partial A_{z}}{\partial t} d \Omega-\int_{\Omega_{j}^{w-}} \frac{\partial A_{z}}{\partial t} d \Omega, G_{p, k}=\int_{\Omega_{p, k}^{b}} \frac{\partial A_{z}}{\partial t} d \Omega,
$$

where $\Omega_{j}^{\mathrm{w}+}$ and $\Omega_{j}^{\mathrm{w}-}$ are the subdomains corresponding the $j$-th coil (the superscript "+" shows that the winding path coincides with the direction of the $z$ axis, and "--" means that the winding path does not coincide with the direction of the $z$ axis); $\Omega_{p, k}^{b}$ is the subdomain corresponding the $k$-th rod of the squirrel cage with a number $p$.

The first differential equation of the system in subdomains corresponding to the air space and magnetic circuit has the zero right-hand side while, in the areas corresponding to the stator and rotor windings, it has the form:

$$
\begin{gathered}
-\frac{1}{\mu_{0}} \Delta A_{z}=\alpha \sigma^{\mathrm{w}}\left(-\frac{l}{l_{b} S^{\mathrm{w}}} Q_{i}+\frac{V_{i}^{-}-V_{i}^{+}}{n_{b} l_{b}}\right) \text { in } \Omega_{i}^{\mathrm{w}+} \text { and } \Omega_{i}^{\mathrm{w}-}, \\
-\frac{1}{\mu_{0}} \Delta A_{z}=\sigma_{p}^{b}\left(\frac{1}{S_{p}^{b}} G_{p, k}+\frac{2 V_{p, k}}{l}\right) \text { in } \Omega_{p, k}^{b},
\end{gathered}
$$

where $\Delta=\nabla \cdot \nabla$ is laplacian operator; $\alpha=1$ in the subdomain $\Omega_{i}^{\mathrm{w}-}$ and $\alpha=-1$ in the subdomain $\Omega_{i}^{\mathrm{w}+} ; V_{i}^{-}$and $V_{i}^{+}$are the values of the scalar electrical potential at the "input" and "output" of the coil with a number $i$.

The hybrid equation system is solved with the use of finite element method and Newton method in order to take into account the nonlinear dependence of the magnetic permeability on the magnetic induction. At each time instant $t$, the code calculates the distribution of the magnetic vectorpotential $A_{z} x, y, t$ in the cross-section of the active volume of the induction motor and the values of the scalar electrical potential $V_{j} t$ at the points where the coils of the parallel branches of the stator winding phase are connected, and where the rods of the rotor squirrel cage are connected to the corresponding short-circuiting rings. 
The distribution of the magnetic potential $A_{z}$ is used to find the distribution of the vector of the magnetic induction in the cross section of the active volume, and this distribution is further used to calculate electromagnetic forces and the rotating torque $M t$. Afterward, taking into account the known load torque $M_{0} t$ of the electric motor, the solution of the ordinary differential equation

$$
J \frac{d \omega}{d t}=M t-M_{0} t
$$

is used to determine the instantaneous rotational velocity of rotor $\omega$ (here, $J$ is the moment of inertia). The obtained value of the rotational velocity $\omega$ is then employed to calculate the angle by which the rotor rotates during the time $\Delta t$, and the corresponding turn is made.

In the process of simulation, the code calculates the instantaneous values of all currents in stator winding coils, rods, and rings of short-circuited rotor windings, thermal losses, consumed power, and accumulated magnetic energy that are used to determine all the required characteristics. In addition, at each moment of time, the distribution of the magnetic field in the entire volume of the motor is consistently calculated with the currents in stator and rotor windings (i.e., the dependences of the currents on the magnetic field fluxes through winding circuits and the dependence of the magnetic field on currents are taken into account).

The simulation process itself may be realized in two variants. In the first case, the rotor rotates with some preset velocity, and in the second case, the rotation velocity is calculated using the electromagnetic force during the process of the simulation of the electromagnetic field. The user can use one or another variant depending on the problem to be solved.

In order to take into account the end-zones influence on the values of the magnetic fluxes and rotating torque at each time step, the nonlinear magnetostatic problem is solved in the $3 \mathrm{D}$ structure of induction motor. In this case, the currents obtained in the rods and short-circuiting rings of the squirrel cages of the rotor and in the stator windings are taken as the sources of the magnetic field. For the solution of the nonlinear $3 \mathrm{D}$ magnetostatic problem, the mathematical model based on the total scalar potential for describing the magnetic field inside the ferromagnetic is used. The numerical scheme and the examples of its applications for calculating the 3D magnetic field in the induction motor are presented in [12].

\section{B. Software}

The structure of the induction motor is designed with the use of the graphical interface during the following stages: designing the rotor and stator slots, generating the 2D model; designing the stator winding, and generating the 3D model.

The design of the rotor and stator slots can be performed in two variants. The user can select the slot configuration from the "slots library" (where in a special format the geometries of some standard slots are kept) and, if necessary, correct its parameters. The user can also design a new slot configuration with special graphic tools and then save this slot configuration into the "slots library".

At the next stage, the 2D model is generated. The user sets the following parameters: the value of the air gap, outer radii of the rotor and stator, as well as the number of slots of the rotor and stator. Based on these parameters, the 2D model of the induction motor and corresponding finite element mesh are generated automatically. They are displayed in the main window of the graphic interface. One of the examples is shown in Fig. 1a.

The design of stator winding and set up one-one correspondence between its components (coils) in the electrical circuit and their space position in the induction motor section is the main stage of designing the induction motor structure for calculating the electrodynamic process with the use of quasi-3D and 3D approaches proposed in [1112]. In order to set the winding parameters, the special window is opened (its view is shown in Fig. 1b). In this window, the user sets the following parameters: the winding type, the number of pairs of poles, the number of phases, the number of winding layers, the value of winding pitch, the number of slots per pole pitch, and the number of slots per pole and phase. As a result, the special data structure is generated. This structure connects the space position of the slot with the "part of winding" passing through it.
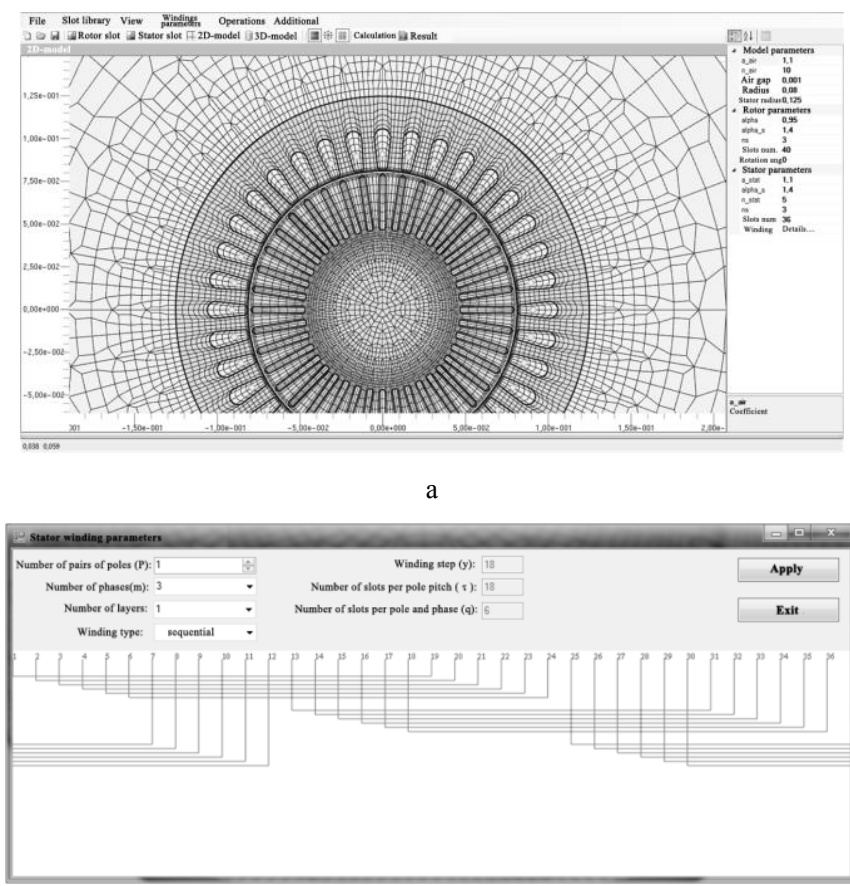

b

Fig. 1. (a) The view of the motor section model with the finite element mesh generated automatically and (b) the window for designing the stator winding

At the last stage, the user sets the length of the motor, and its 3D model with the finite element mesh is generated automatically. The views of 3D induction motor models (shown in perspective) with the finite element meshes in the graphical interface window are shown in Fig. 2 (note that, for clarity, this figure presents only those structural elements 
which correspond to the magnetic circuit, i.e. without a mesh in the windings, rods, short-circuit rings, and air space).

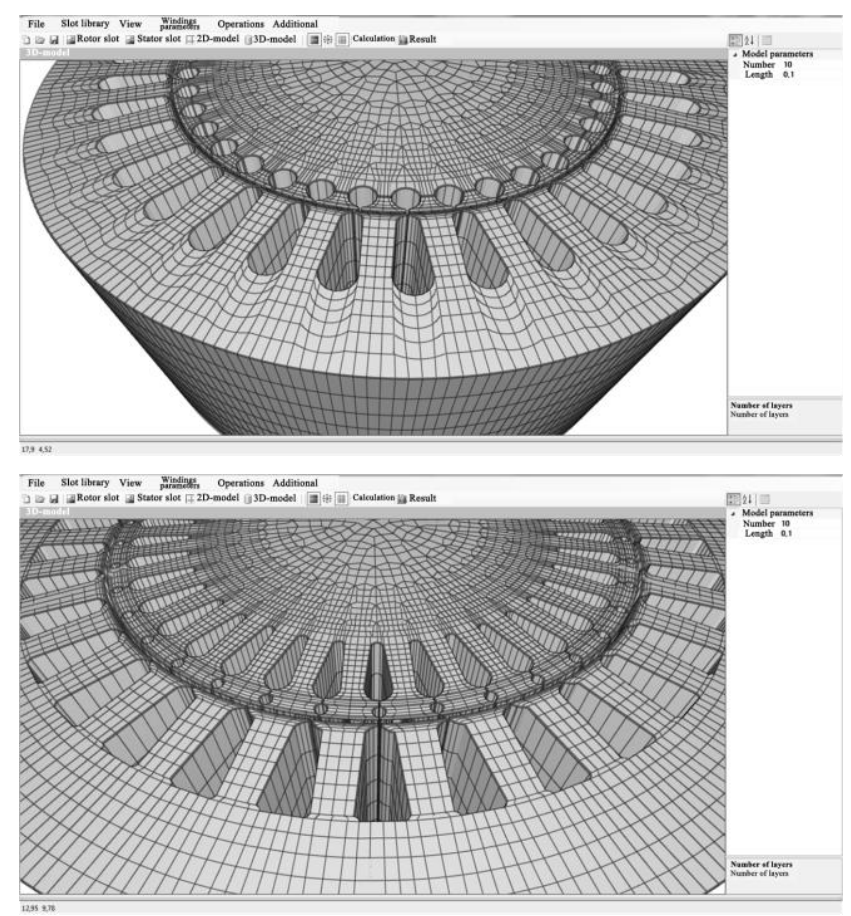

Fig. 2. The view of 3D models of induction motors for different type of slots

\section{COMPARISON OF NUMERICAL RESUlTS WITH EXPERIMENTAL DATA}

In order to validate the proposed approach, the numerical results obtained by the developed code are compared with the experimental data obtained for three-phase double-speed induction motor with the complex geometrical structure of a rotor made as two independent squirrel cages. The 3D view of the part of the induction motor with the finite element mesh and the numerical distribution of the electromagnetic field in the motor section at some time are presented in Fig. 3. Note that Fig. 3a as well as Fig. 2 shows only those structural elements which correspond to the magnetic circuit, i.e. without a mesh in the windings, rods, short-circuit rings, and air space. The two-layered winding of the stator designed for the number of poles $2 p=4$ is located at the bottom of the slot.
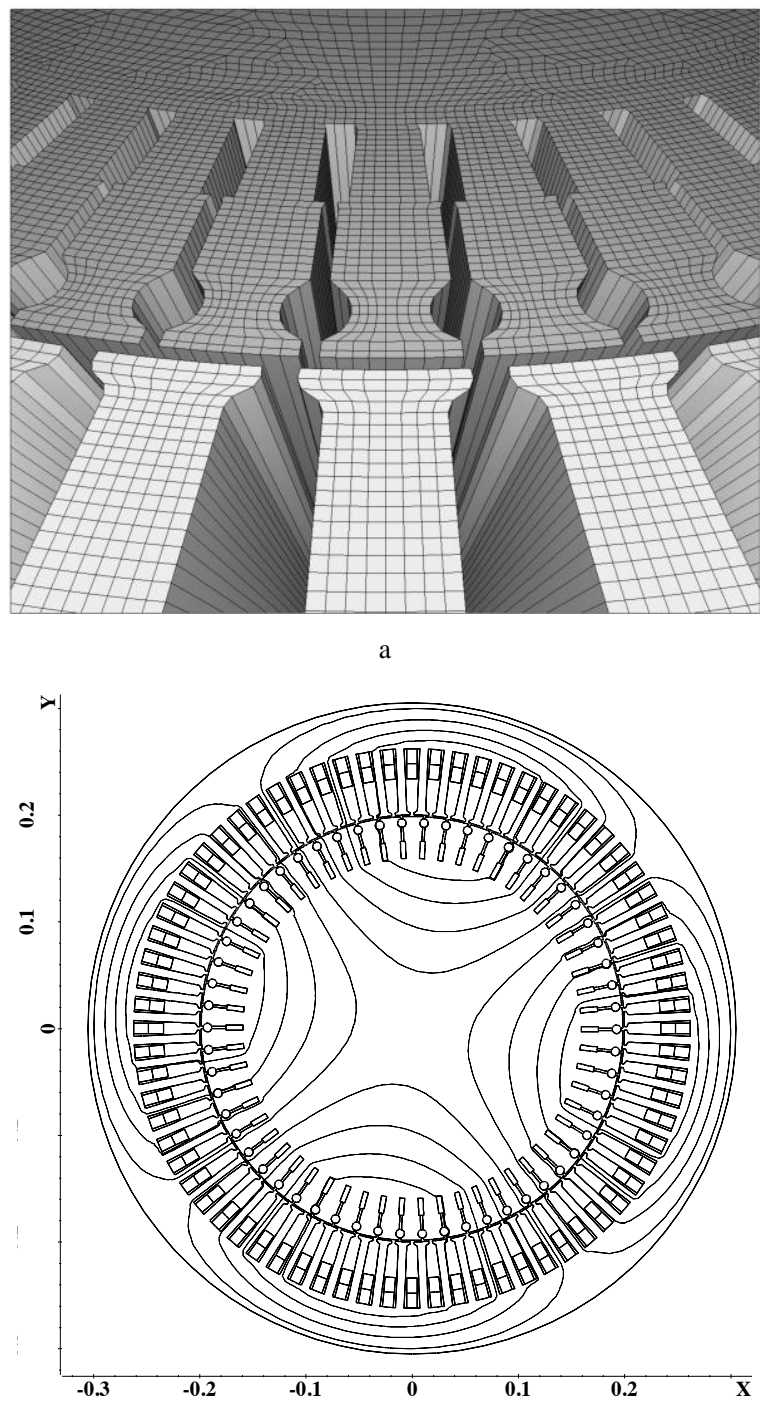

Fig. 3. (a) The 3D view of the induction motor model with the finite element mesh and (b) the numerical distribution of the magnetic field at some time instant in the section of the motor

The simulation was performed for different slip values. The values of the effective current and effective consumed power are compared with the experimental data. Fig.4 shows the effective current and effective consumed power versus the slip, which are obtained by numerical modelling (the corresponding graphs are shown by lines), and experimental data (shown by dots). The experimental data were measured in the range of slip from 1 to $2.22 \%$. The calculated graphs are presented from no-load run to slip value of $3 \%$. The no-load current was $23 \mathrm{~A}$ and the consumed power was $0.4 \mathrm{~kW}$. 


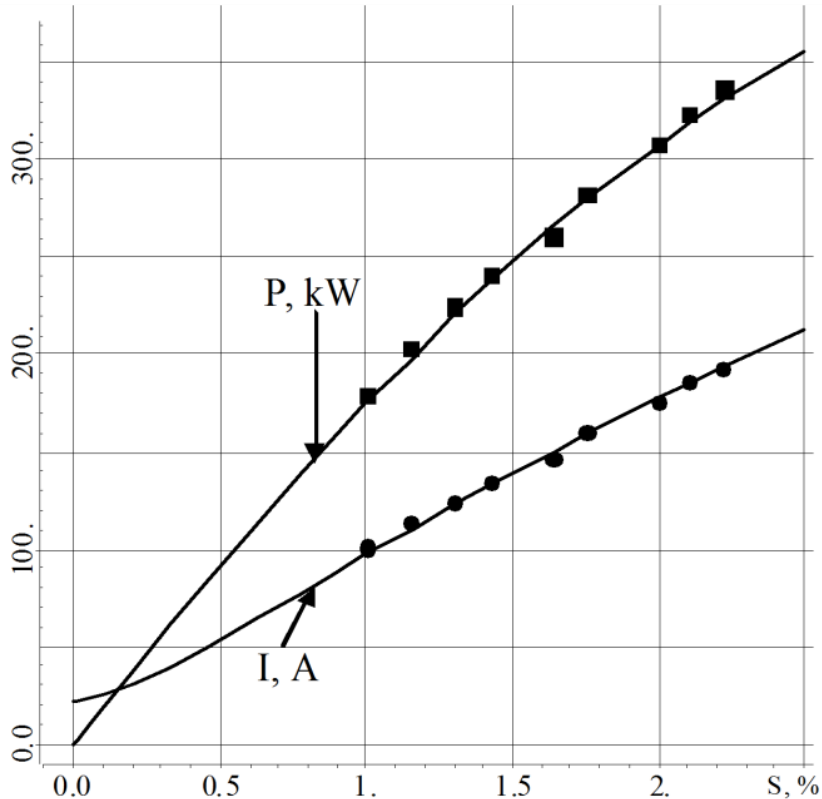

Fig. 4. Experimental (dots) and numerical (lines) effective values of consumed power and current vs slip

The obtained results show that the developed code makes it possible to simulate the electrodynamic processes in induction motors with quite high accuracy.

\section{THE EXAMPlES OF THE DEVELOPED CODE APPLICATIONS}

The possibilities of the developed code are demonstrated by simulating the situations when the motor should accelerate a transport vehicle with some preset values of the mass $m$ to a certain speed. The purpose of the simulation is to analyse the influences of the stator winding voltage frequency on the acceleration process and main characteristics of the induction motor. In this case, the level of the dynamic load is defined by the inertia moment $J$, the value of which was defined with the use of the values of the motor angular velocity $\omega$ and motional energy of a transport vehicle accelerated to the speed $u$ from the relation: $J \omega^{2} / 2=m u^{2} / 2$. The mass $m$ is taken equal to 20 tons, the speed $u$ is taken equal to $60 \mathrm{~km}$ per hour and the corresponding motional energy should be reached when $\omega=314$.

Fig. 5 shows the angular velocity $\omega$ versus time during the acceleration mode of the transport vehicle for different values of frequency $v$ of stator winding voltage $U^{d} \sin 2 \pi v t+\phi_{0}$. The absolute value $U^{d}$ are defined from the relation $U^{d} / v=$ const . Curves $1,2,3,4$, and 5 in Fig. 5a are obtained for the acceleration mode without frequency shift for different values of $v$ and $U^{d}$. Fig. 5b shows the curves 6 , 7 , and 8 which are calculated for the acceleration mode with the frequency shift in the range of $10 \ldots 100 \mathrm{~Hz}$. In this case, the frequency increases by $10 \mathrm{~Hz}$ when the angular velocity $\omega$ approaches the velocity of rotation of the magnetic field in the motor.
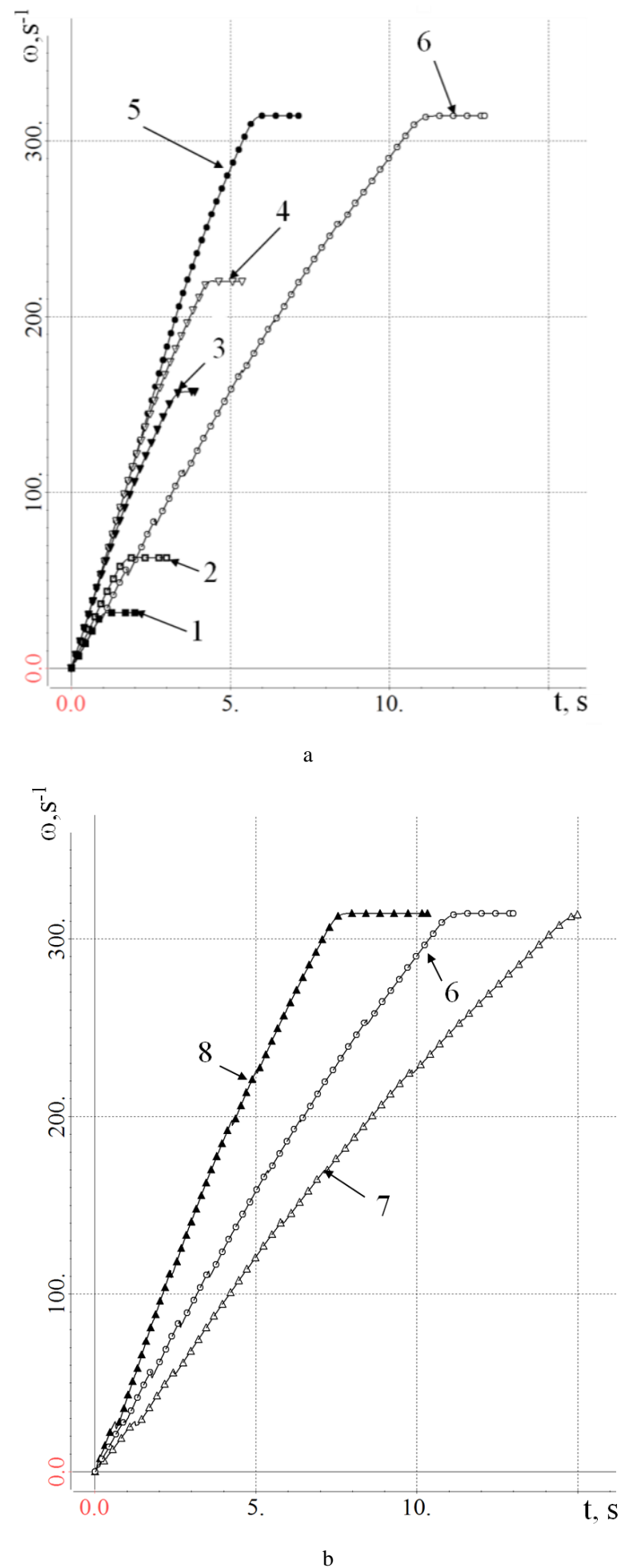

Fig. 5. The values of the angular velocity $\omega$ vs time for the acceleration mode (a) without frequency shift (curve 1 corresponds to $v=10 \mathrm{~Hz}, U^{d}=200 \mathrm{~V}$; curve $2-v=20 \mathrm{~Hz}, U^{d}=400 \mathrm{~V}$; curve $3-v=50 \mathrm{~Hz}, U^{d}=1000 \mathrm{~V}$; curve $4-v=70 \mathrm{~Hz}, U^{d}=1400 \mathrm{~V}$; curve $5-v=100 \mathrm{~Hz}, U^{d}=2000 \mathrm{~V}$ ) and (b) with frequency shift in the range of $10 \ldots 100 \mathrm{~Hz}$ (curve $6-U^{d}$ is changed from $200 \mathrm{~V}$ to $2000 \mathrm{~V}$; curve $7-U^{d}$ is changed from $170 \mathrm{~V}$ to $1700 \mathrm{~V}$; curve $8-U^{d}$ is changed from $240 \mathrm{~V}$ to $2400 \mathrm{~V}$ ) 
As seen from the graphs shown in Fig. 5, during the acceleration, the speed-up grows with the increase of the frequency $v$ of the winding stator voltage (with the corresponding increase in its effective value). However, this does not mean that the mode with fast growing frequency $v$ will be the best one. For more objective comparison, the total thermal losses are analyzed when accelerating to the same speed. The corresponding data are presented in Tables I and II ("-" denotes that for the corresponding mode, the motor is not accelerated to the preset speed; the values of thermal losses are given in $\mathrm{kJ}$ ).

TABLE I. TOTAL THERMAL LOSSES (KJ) FOR MODES WITHOUT FREQUENCY SHIFT

\begin{tabular}{|c|c|c|c|c|}
\hline \multirow{2}{*}{$\omega, \mathrm{s}^{-1}$} & \multicolumn{4}{|c|}{ Mode characteristics } \\
\cline { 2 - 5 } & $\begin{array}{c}v=20 \mathrm{~Hz} \\
U^{d}=400 \mathrm{~V}\end{array}$ & $\begin{array}{c}v=50 \mathrm{~Hz} \\
U^{d}=1000 \mathrm{~V}\end{array}$ & $\begin{array}{c}v=70 \mathrm{~Hz} \\
U^{d}=1400 \mathrm{~V}\end{array}$ & $\begin{array}{c}v=100 \mathrm{~Hz} \\
U^{d}=2000 \mathrm{~V}\end{array}$ \\
\hline 60 & 615 & 1876 & 2700 & 3662 \\
\hline 150 & - & 3145 & 5088 & 7997 \\
\hline 220 & - & - & 5820 & 10106 \\
\hline $\begin{array}{c}\text { Total } \\
\text { acceleration } \\
\omega=314\end{array}$ & - & - & - & 11542 \\
\hline
\end{tabular}

TABLE II. TOTAL THERMAL LOSSES (KJ) FOR MODES WITH FREQUENCY SHIFT

\begin{tabular}{|c|c|c|c|}
\hline \multirow{2}{*}{$\omega, \mathrm{s}^{-1}$} & \multicolumn{3}{|c|}{ Mode characteristics } \\
\cline { 2 - 4 } & $\begin{array}{c}v=10 \ldots 100 \mathrm{~Hz} \\
U^{d}=200 \ldots 2000 \mathrm{~V}\end{array}$ & $\begin{array}{c}v=10 \ldots 100 \mathrm{~Hz} \\
U^{d}=170 \ldots 1700 \mathrm{~V}\end{array}$ & $\begin{array}{c}v=10 \ldots 100 \mathrm{~Hz} \\
U^{d}=240 \ldots 2400 \mathrm{~V}\end{array}$ \\
\hline 60 & 632 & 569 & 690 \\
\hline 150 & 1797 & 1420 & 1875 \\
\hline 220 & 2762 & 2185 & 2971 \\
\hline $\begin{array}{c}\text { Total } \\
\text { acceleration } \\
\omega=314\end{array}$ & 3901 & 3250 & 4186 \\
\hline
\end{tabular}

These data show that total thermal losses of energy, when accelerating to the same speed, markedly grow with the increase in speed-up. This suggests that the optimization of the acceleration mode should be performed taking into account relationship between the reached speed-up and required energy costs, which are accompanied by possible overheating of the motor.

To confirm this, the effective values of currents versus time are considered for acceleration modes with different frequencies of the stator winding voltage. The corresponding graphs are shown in Fig. 6. The legend is the same as in Fig. 5 $(t=1 \mathrm{~s}, 1.8 \mathrm{~s}, 2.65 \mathrm{~s}, 3.55 \mathrm{~s}, 4.5 \mathrm{~s}, 5.4 \mathrm{~s}, 6.4 \mathrm{~s}, 7.4 \mathrm{~s}, 8.4 \mathrm{~s}$ are the time instants which correspond to the time of change of voltage frequency with increment of $10 \mathrm{~Hz}$, i.e. $v=10 \mathrm{~Hz}$ from 0 to $1.8 \mathrm{~s}, v=20 \mathrm{~Hz}$ from $1.8 \mathrm{~s}$ to $2.65 \mathrm{~s}$, etc.). Fig. 7 shows the values of the thermal losses in the stator winding, which are defined by these currents (Fig. $7 a$ ), as well as the values of the total thermal losses in two squirrel cages of the rotor (Fig. 7b). Fig. 7c shows the graphs of the thermal losses in the rotor for the acceleration mode with frequency shift $\left(v=10 \ldots 100 \mathrm{~Hz}, U^{d}=200 \ldots 2000 \mathrm{~V}\right)$. These values are presented separately for the top and bottom squirrel cages.

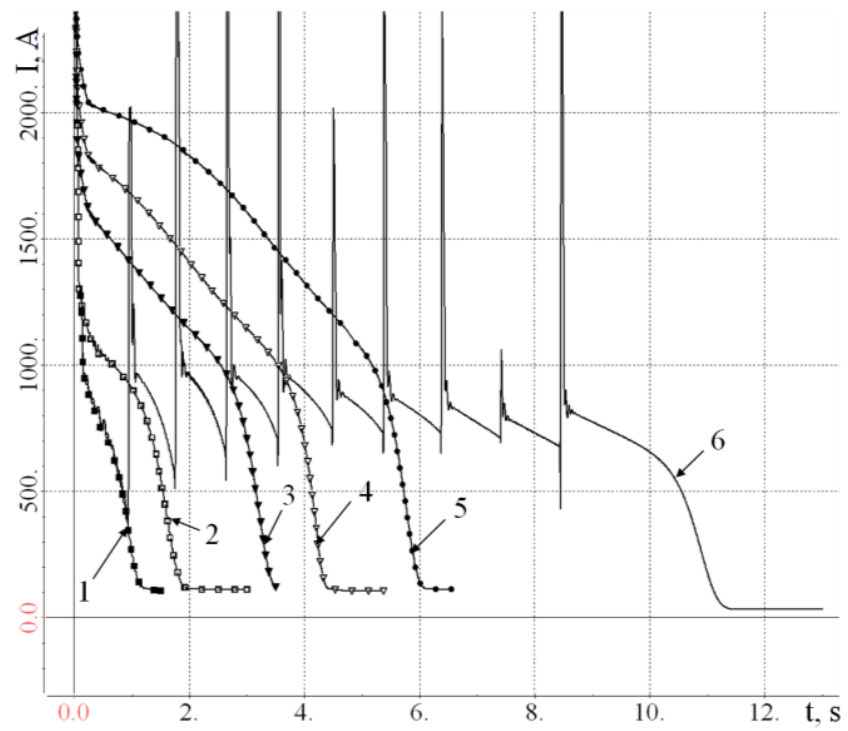

Fig. 6. The effective values of currents vs time for acceleration modes with different frequencies (the legend is the same as in Fig. 5)
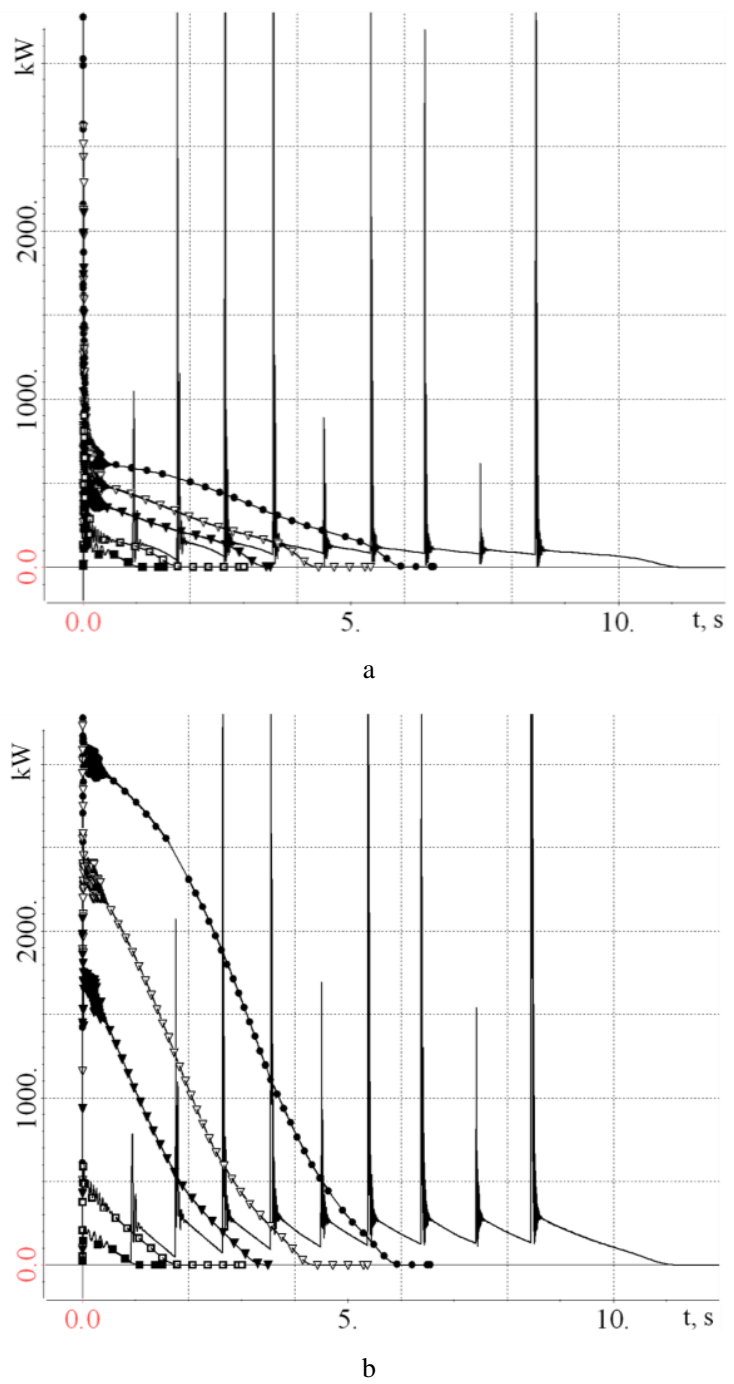


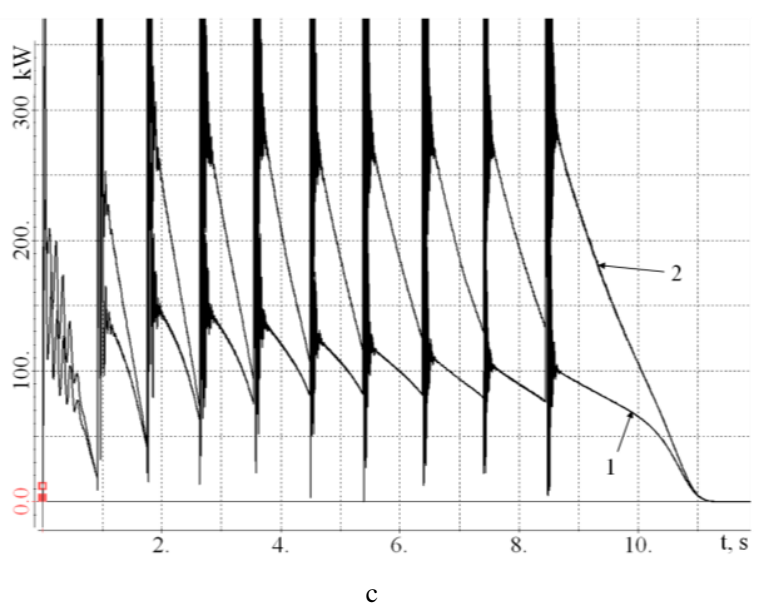

Fig. 7. The values of (a) thermal losses in the stator winding, which are defined by the related currents; (b) the total thermal losses in two squirrel cages of the rotor; (c) thermal losses in the rotor for the acceleration mode with frequency shift; the losses are shown separately for the top and bottom squirrel cages (curve 1 corresponds to the losses in the rods of the bottom cage; curve 2 corresponds to the losses in the rods of the top cage). The curve labels in (a) and (b) are the same as in Fig. 6

\section{CONCLUSIONS}

The developed software makes it possible the fast and convenient design of induction motor structure and the simulation of electrodynamic processes taking into account time-dependent load and stator winding voltage. The proposed software allows us not only to simulate the acceleration processes but also to forecast the occurrence of the emergency state under different operation conditions.

\section{Acknowledgment}

The study was performed with financial support from the Ministry of Education and Science of the Russian Federation under the project part of the state task № 5.978.2017/4.6.

\section{References}

[1] J. Cheaytani, A. Benabou, A. Tounzi, M. Dessoude, L.Chevallier, and T. Henneron, "End-region leakage fluxes and losses analysis of cage induction motors using 3-D finite-element method." IEEE Transactions on Magnetics, vol. 51(3), pp. 1-4, 2015.

[2] G. Lv, Z. Liu, and S. Sun, "Electromagnetism calculation of single-sided linear induction motor with transverse asymmetry using finite-element method.” IET Electric Power Applications, vol. 10(1), pp. 63-73, 2016.

[3] J. Bacher, F. Waldhart, and A. Muetze, "3-D FEM Calculation of Electromagnetic Properties of Single-Phase Induction Machines." IEEE Transactions on Energy Conversion, vol. 30(1), pp. 142-149, 2015.

[4] Y. Xie, and Y. Wang, "3D temperature field analysis of the induction motors with broken bar fault." Applied Thermal Engineering, vol. 66(1), pp. 25-34, 2014

[5] M. Lefik, "Design of permanent magnet synchronous motors including thermal aspects." COMPEL: The International Journal for Computation and Mathematics in Electrical and Electronic Engineering, vol. 34(2), pp. 561-572, 2015.

[6] R. Pechanek, V. Kindl, and B. Skala, "Transient thermal analysis of small squirrel cage motor through coupled FEA." Science Journal, pp. 560-563, 2015.

[7] M. Popescu, D.G. Dorrell, L. Alberti, N. Bianchi, D.A. Staton, and D. Hawkins, "Thermal analysis of duplex three-phase induction motor under fault operating conditions." IEEE Transactions on Industry Applications, vol. 49(4), pp. 1523-1530, 2013.

[8] V.M. Jimenez-Mondragon, R. Escarela-Perez, E. Melgoza, M.A. Arjona and J.C. Olivares-Galvan, "Nonlinear time-harmonic analysis of multiple magnetic field systems: Cartesian, axisymmetric, and coupled circuits.” IEEE Transactions on Magnetics, vol. 52(10), pp. 1-10, 2016.

[9] W. Pietrowski, "3D analysis of influence of stator winding asymmetry on axial flux." COMPEL-The international journal for computation and mathematics in electrical and electronic engineering, vol. 32(4), pp. 1278-1286, 2013

[10] A. Dalcali, and M. Akbaba, "Comparison of 2D and 3D magnetic field analysis of single-phase shaded pole induction motors." Engineering Science and Technology, an International Journal, vol. 19(1), pp. 1-7, 2016.

[11] Z.S. Temlyakova, M.G. Persova, Y.G. Soloveichik, R.V. Petrov, and V.V. Grechkin, "A new approach to designing electrical machines on the basis of numerical simulation.” Russian Electrical Engineering, vol. 78(9), pp. 461-466, 2007.

[12] M.G. Persova, Y.G. Soloveichik, Z.S. Temlyakova, M.V. Abramov, D.V. Vagin, and M.V. Gamadin, Finite element method for modeling three-dimensional nonlinear magnetic fields in electrotechnical devices. Russian Electrical Engineering, vol. 82(6), pp. 292-297, 2011. 